\title{
Induction of apoptosis in human leukemia cells by 3- deazaadenosine is mediated by caspase-3-like activity
}

\author{
Ho-Shik Kim ${ }^{1 *}$, Seong-Yun Jeong ${ }^{1 *}$, \\ Jeong-Hwa Lee ${ }^{1}$, Boe-Eun Kim ${ }^{1}$, Jin Woo Kim², \\ Seong-Whan Jeong ${ }^{1}$ and In-Kyung Kim ${ }^{1,2,3}$ \\ ${ }^{1}$ Department of Biochemistry, College of Medicine, \\ The Catholic University of Korea, Seoul 137-701, Korea \\ ${ }^{2}$ Research Institute of Molecular Genetics, College of Medicine, \\ The Catholic University of Korea, Seoul 137-701, Korea \\ ${ }^{3}$ Corresponding author: Tel, +82-2-590-1175; Fax, +82-2-596-4435; \\ E-mail, ikkim@cmc.cuk.ac.kr
}

Accepted 14 November 2000

Abbreviations: DZA, 3-deazaadenosine; DZA-Hcy; 3-deazaadenosylhomocysteine; Ado, adenosine; Ado-Hcy, S-adenosylhomocysteine; Ado-Met, S-adenosylmethionine; DIG, digoxigenin; DZAri, 3deaza- $( \pm$ )-aristeromycin; Adox; adenosine periodate oxidized; NBTI, nitrobenzylthioinosine; DPD, dipyridamole; CHX, cycloheximide; PARP, poly (ADP-ribose) polymerase

\begin{abstract}
3-Deazaadenosine (DZA), one of the potent inhibitors of S-adenosylhomocysteine hydrolase, is known to possess several biological properties including an induction of apoptosis. To evaluate a possibility that DZA may be utilized for the treatment of human leukemia, we studied molecular events of cell death induced by DZA in human leukemia HL-60 and U-937 cells. DZA induced a specific cleavage of poly ADPribose polymerase (PARP) and an activation of the cysteine protease caspase-3/CPP32 which is known to cleave PARP. DZA-mediated nuclear DNA-fragmentation was completely blocked in the presence of a universal inhibitor of caspases (z-VAD-fmk) or the specific inhibitor of caspase-3 (z-DEVD-fmk) unlike of cycloheximide (CHX). DNA fragmentation was preceded by the lowering of c-myc mRNA in the DZA treated cells. In addition, DZA-induced apoptosis was blocked by pretreatment with adenosine transporter inhibitors such as nitrobenzylthioinosine (NBTI) and dipyridamole (DPD). Taken together, these results demonstrate that DZA-induced apoptosis initiated through an active transport of DZA into human leukemia cells, is dependent on the caspase-3-like activity without de novo synthesis of proteins and
\end{abstract}

${ }^{*}$ These authors contributed equally to this work possibly involves $c$-myc down-regulation.

Keywords: 3-deazaadenosine, leukemia, apoptosis, caspase, c-myc

\section{Introduction}

Apoptosis (i.e., programmed cell death) is known as an important biologic mechanism that contributes to the maintenance of the integrity of multicellular organisms. It is induced by a wide variety of cellular stresses such as DNA damage, UV radiation, ionizing radiation and oxidative stress (Nagata, 1997), and is morphologically distinct from necrosis in many of its characteristic changes as follows; DNA fragmentation, chromatin condensation, cytoplasmic membrane blebbing, and cell shrinkage. Antitumor agents also induce apoptosis in some cancer cells both in vitro and in vivo, indicating that apoptosis plays a very important role in cancer chemotherapy (Kaufmann, 1989; Meyn et al., 1995).

Various stimuli of apoptosis lead to an activation of cytoplasmic cysteine proteases with specificity for aspartic acid residues, namely caspases. The activated caspases can cleave structural proteins and enzymes necessary for the survival of both proliferating and resting cells (Cohen, 1997; Nicholson and Thornberry, 1997; Thornberry and Lazebnik, 1998). In addition, caspases have been shown to activate the endonuclease responsible for the internucleosomal cleavage of genomic DNA, a hallmark of apoptosis (Liu et al., 1997; Enari et al., 1998). An important pathway of cell-death involves the release of cytochrome $c$ from mitochondria to the cytosol, thereby triggering caspase- 3 activation and initiation of the apoptotic program at a relatively late stage (Liu et al., 1996; Kluck et al., 1997; Yang et al., 1997). One important component of the caspase cascade is caspase- 3 which is activated by two sequential proteolytic events that cleave the $32 \mathrm{kD}$ precursor at aspartic acid residues to generate an active heterodimer of 20- and 12 kD subunits (Salvesen and Dixit, 1997). The activation can either be autocatalytic or occur via a caspase cascade.

The protooncogene $c$-myc encoding a nuclear phosphoprotein (c-Myc) of the helix-loop-helix/leucine-zipper family, when dimerized with its binding partner Max, binds to specific DNA sequences (Iguchi-Ariga et al., 1987; Kato and Dang, 1992). Many recent researches on c-Myc have focused on how it drives apoptosis. cMyc is widely known as a crucial regulator of cell 
proliferation in normal and neoplastic cells, but until recently its apoptotic properties, which appear to be intrinsic, were not fully appreciated (Thompson, 1998; Prendergast, 1999).

3-Deazaadenosine (DZA) was developed as one of the potent inhibitors of S-adenosylhomocysteine (Ado-Hcy) hydrolase (EC 3.3.1.1) (Chiang et al., 1977). This agent binds to Ado-Hcy hydrolase resulting in the accumulation of Ado-Hcy and S-adenosylmethionine (Ado-Met), and serves as a substrate for that enzyme resulting in the huge accumulation of 3-deazaadenosylhomocysteine (DZA-Hcy) in cultured cells (Chiang et al., 1977; Aksamit et al., 1982), especially in the liver tissue (Chiang and Cantoni, 1979). DZA exerts a number of interesting biological properties such as anti-human immunodeficiency virus (HIV) activity (Flexner et al., 1992; Mayers et al., 1995), immunosuppressive and anti-inflammatory effects (Medzihradsky et al., 1982; Krenitsky et al., 1986), inhibition of lymphocyte-mediated cytolysis (Zimmerman et al., 1978), inhibition of cytokine expression including tumor necrosis factor- $\alpha$ (TNF- $\alpha$ ) and interleukin-1 $\beta$ (IL$1 \beta)$ (Jeong et al., 1996), inhibition of cell adhesion molecule expression (Jurgensen et al., 1990; Shankar et al., 1992), inhibition of nuclear factor- $\kappa B$ (NF- $\kappa B$ ) transcriptional activity (Jeong et al., 1999), and induction of apoptosis in human and mouse leukemia cells (Endresen et al., 1993; Kim et al., 1997).

Although the potent apoptosis-inducing effect of DZA highlights the possibility that DZA might be applied for the treatment of human leukemia, the mechanism based on molecular events in the DZA-induced human leukemic apoptosis has not been fully elucidated. Thus, to evaluate the possibility, we have investigated the apoptotic mechanism of DZA in human leukemia HL-60 and $\mathrm{U}-937$ cells by determining alterations of apoptosisrelated molecules such as caspase-3 and c-myc.

\section{Materials and Methods}

\section{Materials and reagents}

DZA and 3-deaza- $( \pm)$-aristeromycin (DZAri) were donated by Dr. Chiang of the Walter Reed Army Institute of Research, Washington DC., and inhibitors of caspases were obtained from Bio-Rad Laboratories (Hercules, CA). Mouse anti-poly(ADP-ribose) polymerase was obtained from Serotec (Oxford, UK) and anticaspase-3 $(\mathrm{H}-277)$ was purchased from Santa Cruz Biotechnology (Santa Cruz, CA). Unless specified otherwise, all reagents were purchased from Sigma Chemical Co. (St. Louis, MO).

\section{Cell culture}

Human promyelocytic leukemia HL-60 and human monocytic macrophage U-937 cells were obtained from the
American Type Culture Collection (ATCC, Rockville, MD). Both cells were cultured in RPMI 1640 medium supplemented with $20 \mathrm{mM} \mathrm{N}$-(2-hydroxyethyl) piperazine-N'-2ethanesulphonic acid (HEPES), $25 \mathrm{mM}$ sodium bicarbonate, 100 units $/ \mathrm{ml}$ penicillin, $100 \mu \mathrm{g} / \mathrm{ml}$ streptomycin sulfate and $10 \%$ heat-inactivated $\left(56^{\circ} \mathrm{C}\right.$ for $\left.30 \mathrm{~min}\right)$ fetal bovine serum (Hyclone Laboratories Inc., Logan, UT) at $37^{\circ} \mathrm{C}$ in an atmosphere of $5 \% \mathrm{CO}_{2}$.

\section{Determination of cell death}

Induction of apoptosis was determined by fragmentation of genomic DNA through agarose gel electrophoresis. Cells were treated with DZA or DZA plus other compounds for desired times, and then genomic DNA was extracted using DNA extraction buffer [5 mM Tris-Cl pH 8.0, $20 \mathrm{mM}$ EDTA pH 8.0, 1\% sodium dodecyl sulfate (SDS), $50 \mu \mathrm{g} / \mathrm{ml}$ proteinase $\mathrm{K}]$ and extraction with phenolethanol. DNA pellet was dissolved in TE buffer $(10 \mathrm{mM}$ Tris-Cl pH 8.0, 1 mM EDTA pH 8.0) containing $20 \mu \mathrm{g} / \mathrm{ml}$ of RNase $\mathrm{A}$, and incubated at $37^{\circ} \mathrm{C}$ for $1 \mathrm{~h}$. Five micrograms of DNA was separated on $2 \%$ agarose gels with 0.5x TAE (20 mM Tris-acetate, $1 \mathrm{mM}$ EDTA). Ethidium bromide (EtBr)-stained DNA in the gel was visualized under UV light and photographed. The ratio of DZAinduced cell death was evaluated by cell counting with hemocytometer. Cells with intact cytoplasmic membrane and diameter within the range of control cells were judged as viable cells.

\section{Western blot analysis}

Cells after treatments were harvested and lysed with $2 x$ SDS-polyacrylamide gel electrophoresis (SDS-PAGE) loading buffer. Aliquots of total cell lysates were subjected to $10 \%$ SDS-PAGE for PARP or $12.5 \%$ SDSPAGE for caspase-3 proteins, and transferred to a nitrocellulose membrane in transfer buffer (25 mM Tris base, $193 \mathrm{mM}$ glycine, 20\% methanol). The membrane was blocked in $5 \%$ nonfat dried milk in phosphate-buffered saline (PBS) and then incubated with primary antibody in blocking solution at room temperature for $2 \mathrm{~h}$, followed by extensive washing in PBS containing $0.1 \%$ Tween20. The blot was then incubated with peroxidase-conjugated secondary antibody in blocking solution, followed by washing in PBS containing $0.1 \%$ Tween-20. Antigen was detected using the enhanced chemiluminescence western blotting detection system (Amersham-Pharmarcia, Buckinghamshire, UK).

\section{Northern blot analysis}

Total cellular RNA was isolated from cells treated with DZA according to the previously described method (Chomczynski and Sacchi, 1987). Five micrograms of total RNA was separated on $1 \%$ agarose gels containing 2.2 M formaldehyde. After electrophoresis RNA was transferred to a nylon membrane (Boehringer Mannheim, 
Mannheim, Germany) by capillary transfer in the presence of 20x SSC. Then the filter which was UVcrosslinked with UV-stratalinker 1800 (Stratagene, La Jolla, CA) was prehybridized and hybridized with Digoxigenin (DIG; Boehringer Mannheim, Mannheim, Germany) labeled probe in high SDS hybridization buffer at $50^{\circ} \mathrm{C}$ overnight. DIG-labeled probes were prepared from cloned cDNA by polymerase chain reaction (PCR). After hybridization the nylon membrane was rinsed with $2 x$ SSC, $0.1 \%$ SDS followed by $0.1 \times$ SSC, $0.1 \%$ SDS and hybridized probe was detected by chemiluminescent method with anti-DIG Fab fragments alkaline phosphatase-conjugated (Boehringer Mannheim) and CSPD (Boehringer Mannheim), a substrate of alkaline phosphatase.

\section{Results}

\section{DZA induces apoptosis in human leukemia cells}

To estimate the apoptosis-inducing effect of DZA, human leukemia HL-60 and U-937 cells were treated with 100 $\mu \mathrm{M}$ of DZA for various times. After treatment of DZA, genomic DNA was isolated and subjected to agarose gel electrophoresis. As shown in Figure 1, the treatment of $100 \mu \mathrm{M}$ of DZA led to the internucleosomal cleavage of genomic DNA very rapidly in both cells resulting in DNA ladder in agarose gel electrophoresis that is the major evidence of apoptosis, indicating DZA potently induces apoptosis in human leukemia cells. The induction of apoptosis in HL-60 cells was more rapid and potent than that in U-937 cells. The death proportion

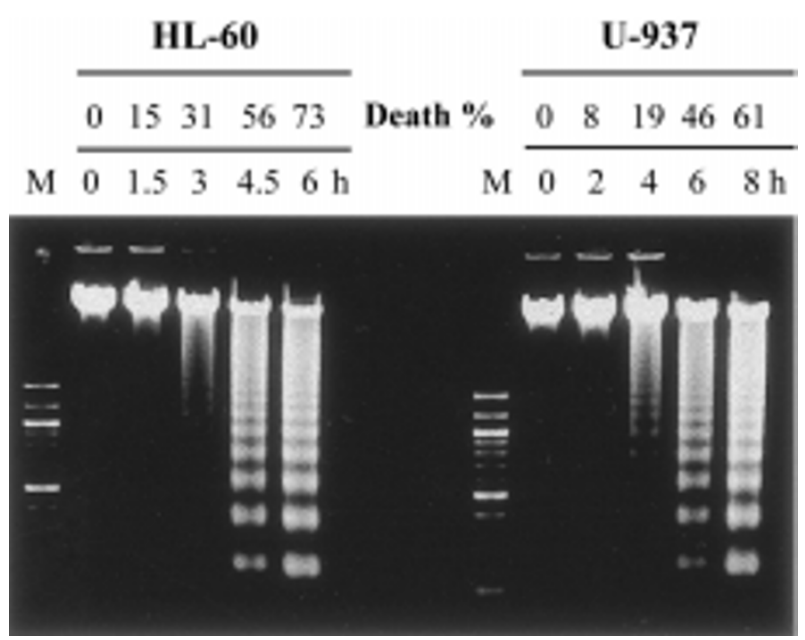

Figure 1. DZA induces cell death with DNA fragmentation in human leukemia cells. Cells were treated with $100 \mu \mathrm{M}$ of DZA for the indicated times. Genomic DNA was extracted as described in 'Materials and Methods', and then $5 \mu \mathrm{g}$ of DNA was separated into $2 \%$ agarose gel. Death $\%$ was determined by cell counting with a hemocytometer. M: molecularweight marker.

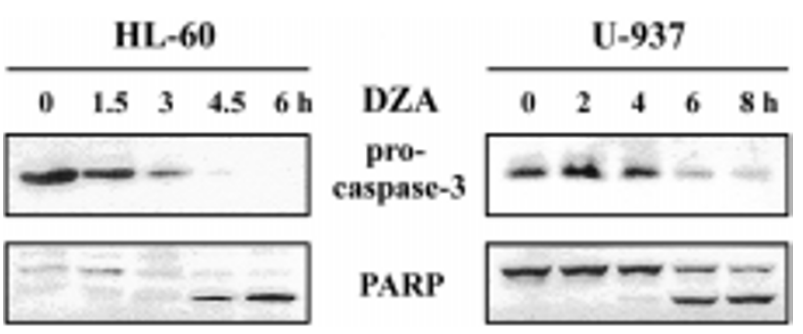

Figure 2. Caspase-3 activation by DZA. Cells were treated with $100 \mu \mathrm{M}$ of DZA for the indicated times. Cell lysate was prepared with $2 x$ SDS-PAGE sample buffer and then Western blot analysis was performed with specific antibodies against pro-caspase-3 and PARP as described in 'Materials and Methods'.

compared to control cells were evaluated as described in materials and methods, and indicated in Figure 1.

\section{Activation of caspase-3-like enzymes is essential in DZA-induced apoptosis}

To determine whether caspase-like activities including caspase-3 are involved in human leukemic apoptosis induced by DZA, we first examined the cleavage of procaspase-3 during DZA-induced apoptosis in HL-60 and U-937 cells. After cells were treated with $100 \mu \mathrm{M}$ of DZA to induce apoptosis as indicated in time-courses, total cell lysates were prepared with $2 \times$ SDS-PAGE loading buffer and then Western blot analysis was performed with these lysates using specific antibodies to caspase-3 and PARP (Figure 2). In both cells, procaspase-3 was gradually reduced as time passed in the presence of DZA. PARP, the substrate of caspase-3 was also cleaved corresponding to the cleavage of procaspase-3 during DZA-induced apoptosis in these cells. These results show that caspase- 3 is activated and its substrate PARP is also processed in apoptotic human leukemia cells induced by DZA. We used inhibitors of caspases in order to determine whether the activation of caspase-3 is necessarily involved in the induction of apoptosis by DZA. Z-VAD-fmk, a general inhibitor of caspases completely blocked the formation of DNA fragmentation mediated by the treatment of DZA in both HL-60 and U-937 cells (Figure 3a), proving that caspase-like activities are involved in this induction of apoptosis. Z-DEVD-fmk, a specific inhibitor of caspase3-like enzymes totally abolished the DNA fragmentation induced by the treatment of DZA, indicating that the caspase-3-like activity is necessary to DZA-induced human leukemic cellular apoptosis (Figure 3a). When the cleavages of genomic DNA induced by DZA in these cells were blocked by inhibitors of caspases, neither procaspase-3 nor its substrate PARP was reduced (Figure $3 b$ ). The observation under the light-microscope revealed that the morphological changes of apoptosis induced by DZA in both cells were not shown in the cells pretreated with inhibitors of caspases (data not shown). 
a

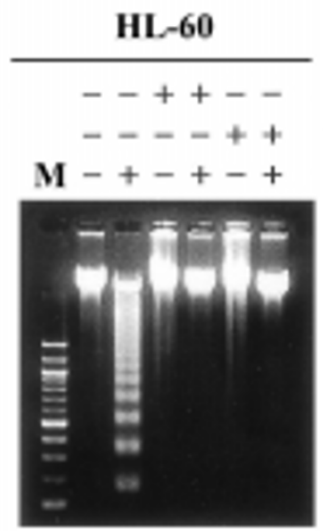

b

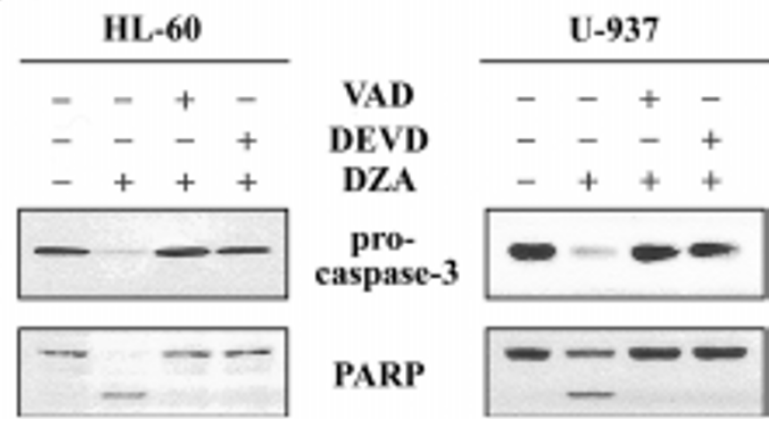

Figure 3. Inhibition of DZA-induced apoptosis by caspase inhibitors. Cells were pretreated with caspase inhibitors $(100 \mu \mathrm{M}$ of $z$-VAD-fmk or $100 \mu \mathrm{M}$ of z-DEVD-fmk) for $1 \mathrm{~h}$ and then treated with $100 \mu \mathrm{M}$ of DZA for $4.5 \mathrm{~h}$ (HL60 ) or $6 \mathrm{~h}$ (U-937). (a) Genomic DNA was extracted and separated into $2 \%$ agarose gel. (b) Cell lysate was prepared with $2 x$ SDS-PAGE sample buffer and then Western blot analysis was performed with specific antibodies against pro-caspase-3 or PARP.

These results indicate that the caspase activity, especially caspase-3-like activity, is necessary to induce apoptosis by DZA in human leukemia cells.

\section{c-myc and puf are down-regulated during DZA- induced apoptosis}

The alteration of $c-m y c$ was shown to be involved in the apoptosis of HL-60 cells (Kimura et al., 1995) and U-937 cells (Bergan et al., 1996) and the expression of c-myc was diminished during adenosine-induced apoptosis in HL-60 cells (Hong et al., 1997). Possible relationships with the c-myc expression with DZA-induced apoptosis in both human leukemia HL-60 and U-937 cells were explored. The expression of puf, a transcriptional regulator of $c$-myc was also examined. After cells were treated with $100 \mu \mathrm{M}$ of DZA for various times, total RNA was extracted and subjected to Northern blot analysis using DIG-labeled c-myc and puf probe. As shown in Figure 4, the expressions of $c$-myc and puf mRNA were dramatically decreased when cells underwent apoptosis by DZA in both cells. The decrease of $c-m y c$ was faster

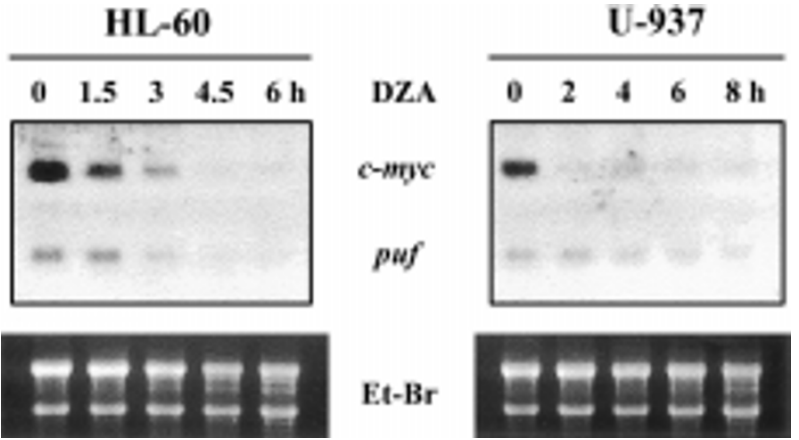

Figure 4. Down-regulation of $c-m y c$ and puf by DZA. Cells were treated with $100 \mu \mathrm{M}$ of DZA for the indicated times. Total RNA was extracted and separated into $1 \%$ agarose/ formaldehyde gel. Northern blot analysis was performed with DIG-labeled c-myc or puf probes as described in 'Materials and Methods'.

a

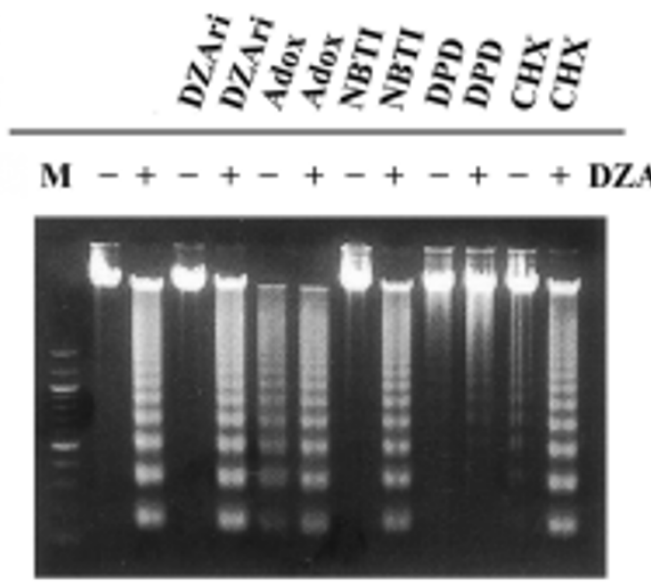

HL-60

b
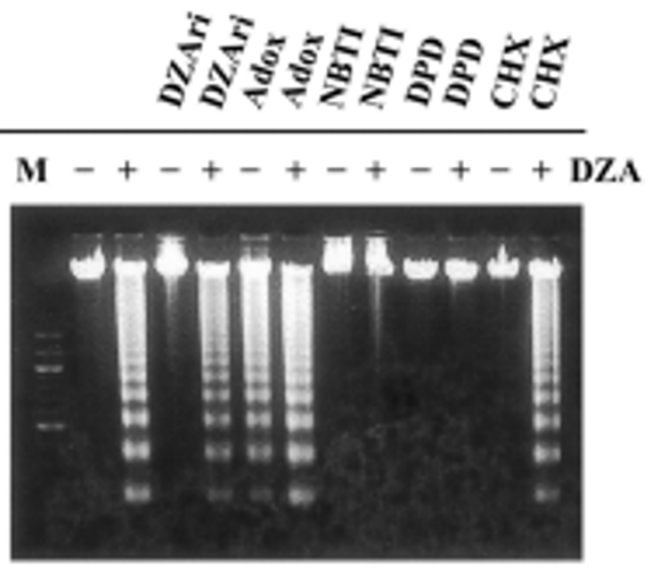

U-937

Figure 5. The effects of Ado transporter inhibitors on the DZA-induced apoptosis. Cells were pretreated with the compounds indicated (DZAri; 200 $\mu \mathrm{M}$ of 3-deaza- $( \pm)$-aristeromycin, Adox; $100 \mu \mathrm{M}$ of adenosine periodate oxidized, NBTI; $10 \mu \mathrm{M}$ of nitrobenzylthioinosine, DPD; $100 \mu \mathrm{M}$ of dipyridamole, $\mathrm{CHX} ; 1 \mu \mathrm{g} / \mathrm{ml}$ of cycloheximide) for $1 \mathrm{~h}$ and then treated with $100 \mu \mathrm{M}$ of DZA for $6 \mathrm{~h}$ (a; HL-60) or $8 \mathrm{~h}$ (b; U-937). Genomic DNA was extracted and separated into $2 \%$ agarose gel. 
than the induction of DNA fragmentation suggesting that the decrease of $c$-myc mRNA expression might be associated with the apoptosis induced by DZA in human leukemia cells.

\section{The effects of adenosine transporter inhibitors on DZA-induced apoptosis}

It has been reported that when cells are treated with $D Z A, D Z A$ is catalyzed to 3-deazaadenosylhomocysteine (DZA-Hcy) and it is largely accumulated in cells (Chiang et al., 1977; Aksamit et al., 1982) as well as in HL-60 cells (Aarbakke et al., 1985). To determinate whether this accumulation of DZA-Hcy is linked to the DZAinduced apoptosis in human leukemia cells, we used 3deaza-( \pm )-aristeromycin (DZAri) (Endresen et al., 1996) and adenosine periodate oxidized (Adox) (Zimmerman et al., 1984) which have been known to be inhibitors of $S$-adenosylhomocysteine hydrolase and block the conversion of DZA to DZA-Hcy. As shown in Figure 5, DZAri and Adox failed to inhibit the apoptosis induced by DZA in HL-60 cells. Similarly, DZAri did not block DZA-induced apoptosis in U-937 cells. Adox brought about apoptosis by itself implying that there may be another effect to induce apoptosis within Adox in HL-60 cells and U-937 cells. These results show that the accumulation of intracellular DZA-Hcy is not required for the induction of apoptosis by DZA in human leukemia cells. Additionally we used inhibitors (Deckert et al., 1988) of the Ado transporter such as dipyridamole (DPD) and nitrobenzylthioinosine (NBTI) in order to investigate whether DZA initiates apoptosis in these cells through active transportation. All of Ado transporters in cancer cells were reported to be sensitive to DPD unlike NBTI (Deckert et al., 1988). Although DZA-induced apoptosis in HL-60 cells was not inhibited by NBTI, the apoptosis was completely inhibited by DPD (Figure 5a). In U-937 cells, both inhibitors totally blocked DZA-induced apoptosis (Figure $5 b$ ). These results revealed that DZA is actively transported into cells by Ado transporters in human leukemic cells. In HL-60 cells there are no NBTIsensitive Ado transporters and therfore NBTI did not inhibit the apoptosis induced by DZA transported through NBTI-insensitive Ado transporters. To establish whether de novo synthesis of proteins is involved in the DZAinduced apoptosis in human leukemia cells, we treated cells with cycloheximide ( $\mathrm{CHX})$. Exposure to $\mathrm{CHX}$ did not diminish the apoptosis induced by DZA in both cells, indicating that de novo synthesis of proteins is not required for the induction of apoptosis by DZA in these cells.

\section{Discussion}

Although the apoptosis-inducing activity of DZA has been suggested as a possible agent for the treatment of human diseases such as leukemia (Sherman et al., 1985; Endresen et al., 1993), the mechanism based on the molecular level has not been clearly elucidated. In this study, we have investigated molecular events of the DZA-induced apoptosis in human leukemia cells in an effort to evaluate DZA as an potential drug.

Among members of the caspase family, caspase- 3 is an effector caspase which plays a role of an executioner in the apoptotic pathway. Human leukemia HL-60 and U-937 cells treated with DZA underwent apoptosis and the activation of caspase-3. The apoptosis was completely blocked by inhibitors of caspases, especially caspase3. Additional treatment of cells with $\mathrm{CHX}$ failed to eliminate DZA-induced apoptosis, indicating this induction of apoptosis doesn't require the expression of additional proteins, but requires the activation of caspase cascade. Considering these results, DZA-induced apoptosis in human leukemia cells appears to be closely linked with the activation of caspase cascade.

c-Myc, a member of the Myc family proteins, regulates cell proliferation and apoptosis. Reduction of $c$-myc expression and its inappropriate expression can be associated with cellular apoptosis (Thompson, 1998). It has been reported that $c$-myc mRNA levels can be reduced by the use of a short antisense oligonucleotide and this reduction is sufficient to initiate apoptosis in $\mathrm{HL}-60$ (Dean et al., 1988; Kimura et al., 1995) and U-937 cells (Bergan et al., 1996). Regarding these reports, the rapid disappearance of $c$-myc during DZA-induced apoptosis in human leukemia HL-60 and U-937 cells indicates that the reduction of $c-m y c$ expression is associated with the induction of this apoptosis. Although inhibitors of caspases abolished DZA-induced apoptosis in these cells, they did not recover the reduced level of $c-m y c$ expression (data not shown). Thus, reduction of $c$-myc might activate caspases or be upstream of caspase cascade in the apoptosis induced by DZA in human leukemia cells. But, there was a discrepancy between $c$-myc downregulation and apoptosis induced by DZA in that whereas $c-m y c$ was down-regulated more rapidly in U-937 cells than HL-60 cells, DNA fragmentation and caspase3 activation were induced more easily in $\mathrm{HL}-60$ cells than U937 cells. Therefore, it can not exclude the possibility that $c$-myc down-regulation is an another effect of DZA not associated with the apoptosis or not the critical event in the DZA-induced apoptosis, and more extensive experiments including over-expression and knockout studies should be performed to assess the role of $c$ $m y c$ in detail.

According to the results of this study, the accumulation of intracellular DZA-Hcy is not required for the induction of apoptosis by DZA in human leukemia cells. We previously reported that DZA inhibited lipopolysaccharide (LPS)-induced NF- $\mathrm{B} B$ transcriptional activity through hindrance of p65 (Rel-A) phosphorylation by the accumulation of DZA-Hcy in mouse macrophage cells 
treated with DZA (Jeong et al., 1999). It has been well known that NF- $\mathrm{kB}$ has an anti-apoptotic property (Antwerp et al., 1996; Beg and Baltimore, 1996; Wang et al., 1996). Additionally, c-myc transcription which is markedly reduced at the early stage of apoptosis induced by DZA in HL-60 and U-937 cells, is a downstream target of NF-kB (Duyao et al., 1990). It has also been previously reported that treatment with $100 \mu \mathrm{M}$ of DZA resulted in significant accumulation of DZA-Hcy in HL60 cells (Aarbakke et al., 1985). Although DZA-Hcy accumulated by the treatment of DZA doesn't directly mediate this induction of apoptosis, an inhibition of NF$\kappa B$ transcriptional activity by the accumulation of DZAHcy might co-operate to induce apoptosis by DZA.

We have determined that DZA-induced apoptosis in human leukemia cells is initiated through the active transport of DZA into cells. Since CHX could not block the DZA-induced apoptosis in human leukemia cells, it appears that up-regulated genes are not as important in the induction of apoptosis by DZA as down-regulated genes such as c-myc.

The apoptosis-inducing activity of DZA shown in this experiment was greater than those of other anti-leukemic drugs such as daunorubicin, mitoxantrone, etoposide and Ara-C (data not shown), although the concentration of DZA, $100 \mu \mathrm{M}$, was the highest among those of the tested drugs. Following the administration of DZA to culture cells or animals, the uptake of DZA was found to be rapid and the net result was the accumulation of Ado-Hcy and DZA-Hcy (Im et al., 1979; Pritchard et al., 1982; Wiesmann et al., 1985) in tissues or organs. Thus, it may be difficult to measure the concentration or maintain the high concentration of administered DZA in blood. The extending pharmacokinetic modification of DZA should be developed to maintain the concentration of DZA at $100 \mu \mathrm{M}$ in blood to utilize it as an effective anti-leukemia drug.

In conclusion, when human leukemia cells are exposed to DZA, it is actively transported into cells, reduces the level of c-myc mRNA, activates caspase cascade, and finally induces cell death by apoptosis without de novo synthesis of proteins. Our results support the possibility that DZA might be an effective drug for the treatment of human leukemia.

\section{Acknowledgements}

The authors wish to acknowledge the financial support of the Korea Research Foundation provided in the program year 1998.

\section{References}

Aarbakke, J., Miura, G. A., Prytz, P. S., Bessesen, A. and Chiang, P. K. (1985) Effects of 3-deazaadenosine on human promyelocytic HL-60 cells. Eur. Paediatr. Haematol. Oncol. 2: 189-192

Aksamit, R. R., Falk, W. and Cantoni, G. L. (1982) Inhibition of chemotaxis by S-3-deazaadenosylhomocysteine in a mouse macrophage cell line. J. Biol. Chem. 257: 621-625

Antwerp, D. J. V., Martin, S. J., Kafri, T., Green, D. R. and Verma, I. M. (1996) Suppression of TNF-alpha-induced apoptosis by NF-кB. Science 274: 787-789

Beg, A. A. and Baltimore, D. (1996) An essential role for NF-KB in preventing TNF- $\alpha$-induced cell death. Science 274: 782-784

Bergan, R., Hakim, F., Schwartz, G. N., Kyle, E., Cepada, R., Szabo, J. M., Fowler, D., Gress, R. and Neckers, L. (1996) Electroporation of synthetic oligodeoxynucleotides: a novel technique for ex vivo bone marrow purging. Blood 88: 731-741

Burns, R. F. (1980) Adenosine receptor activation in human fibroblasts: Nucleoside agonists and antagonists. Can. J. Physiol. Pharmacol. 58: 673-691

Chiang, P. K. and Cantoni, G. L. (1979) Perturbation of biochemical transmethylations by 3-deazaadenosine in vivo. Biochem. Pharmacol. 28: 1897-1902

Chiang, P. K., Richards, H. H. and Cantoni, G. L. (1977) SAdenosyl-L-homocysteine hydrolase: analogues of S-adenosyl-L-homocysteine as potential inhibitors. Mol. Pharmacol. 13: 939-947

Chomczynski, P. and Sacchi, N. (1987) Single-step method of RNA isolation by acid guanidinium thiocyanate-phenol-chloroform extraction. Anal. Biochem. 162: 156-159

Cohen, G. M. (1997) Caspases: the executioners of apoptosis. Biochem. J. 326: 1-16

Dean, M., Cleveland, J., Kim, H. Y., Campisi, J., Levine, R. A., Ihle, J. N. and Rapp, U. (1988) Deregulation of the c-myc and $\mathrm{N}$-myc genes in transformed cells. Curr. Top. Microbiol. Immunol. 141: 216-222

Deckert, J., Morgan, P. F. and Marangos, P. J. (1988) Adenosine uptake site heterogeneity in the mammalian CNS? Uptake inhibitors as probes and potential neuropharmaceuticals. Life Sci. 42: 1331-1345

Duyao, M. P., Buckler, A. J. and Sonenshein, G. E. (1990) Interaction of an NF-kappa B-like factor with a site upstream of the c-myc promoter. Proc. Natl. Acad. Sci. USA 87: 4727-4731

Enari, M., Sakahira, H., Yokoyama, H., Okawa, K., Iwamatsu, A. and Nagata, S. (1998) A caspase-activated DNase that degrades DNA during apoptosis, and its inhibitor ICAD. Nature 391: $43-50$

Endresen, P. C., Eide, T. J. and Aarbakke, J. (1993) Cell death initiated by 3 -deazaadenosine in HL-60 cells is apoptosis and is partially inhibited by homocysteine. Biochem. Pharmacol. 46: 1893-1901

Endresen, P. C., Loennechen, T., Kildalsen, H. and Aarbakke, J. (1996) Apoptosis and transmethylation metabolites in HL60 cells. J. Pharmacol. Exp. Ther. 278: 1318-1324

Flexner, C. W., Hildreth, J. E., Kuncl, R. W. and Drachman, D. B. (1992) 3-Deaza-adenosine and inhibition of HIV. Lancet 339: 438 
Hong, S. H., Jeong, S. Y., Ahn, S. G., Hwang, I. S. and Kim, I. K. (1997) Active adenosine transporter-mediated adenosine entrance into HL-60 cells leads to the induction of apoptosis through down-regulation of c-Myc. Exp. Mol. Med. 29(3): 183-190

Iguchi-Ariga, S. M., Itani, T., Kiji, Y. and Ariga, H. (1987) Possible function of the c-myc product: promotion of cellular DNA replication. EMBO J. 6: 2365-2371

Im, Y. S., Chiang, P. K. and Cantoni, G. L. (1979) Guanidoacetate Methytransferase. J. Biol. Chem. 254:11047-11050

Jeong, S. Y., Ahn, S. G., Lee, J. H., Kim, H. S., Kim, J. W., Rhim, H., Jeong, S. W. and Kim, I. K. (1999) 3-deazaadenosine, a S-adenosylhomocysteine hydrolase inhibitor, has dual effects on NF- $\kappa B$ regulation. Inhibition of NF- $\kappa B$ transcriptional activity and promotion of IkappaBalpha degradation. J. Biol. Chem. 274: 18981-18988

Jeong, S. Y., Lee, J. H., Kim, H. S., Hong, S. H., Cheong, C. H. and Kim, I. K. (1996) 3-Deazaadenosine analogues inhibit the production of tumour necrosis factor-alpha in RAW264.7 cells stimulated with lipopolysaccharide. Immunology 89: 558-562

Jurgensen, C. H., Huber, B. E., Zimmerman, T. P. and Wolberg, G. (1990) 3-deazaadenosine inhibits leukocyte adhesion and ICAM-1 biosynthesis in tumor necrosis factor-stimulated human endothelial cells. J. Immunol. 144: 653-661

Kato, G. J. and Dang, C. V. (1992) Function of the c-Myc oncoprotein. FASEB J. 6: 3065-3072

Kaufmann, S. H. (1989) Induction of endonucleolytic DNA cleavage in human acute myelogenous leukemia cells by etoposide, camptothecin, and other cytotoxic anticancer drugs: a cautionary note. Cancer Res. 49: 5870-5879

Kim, I.-K., Li, C.-C. H., Young, H. A., Lee, J.-H., Kim, H.-S., Pardhasaradhi, K., Garcia, G. E. and Chiang, P. K. (1997) Apoptosis of L1210 leukemia cells induced by 3-deazaadenosine analogs: Differential expression of c-myc, NF-KB and molecular events. J. Biomed. Sci. 4: 83-90

Kimura, S., Maekawa, T., Hirakawa, K., Murakami, A. and Abe, T. (1995) Alterations of c-myc expression by antisense oligodeoxynucleotides enhance the induction of apoptosis in $\mathrm{HL}-60$ cells. Cancer Res. 55: 1379-1384

Kluck, R. M., Bossy-Wetzel, E., Green, D. R. and Newmeyer, D. D. (1997) The release of cytochrome c from mitochondria: A primary site for $\mathrm{Bcl}-2$ regulation of apoptosis. Science 275 : 1132-1136

Krenitsky, T. A., Rideout, J. L., Chao, E. Y., Koszalka, G. W., Gurney, F., Crouch, R. C., Cohn, N. K., Wolberg, G. and Vinegar, R. (1986) Imidazo[4,5-c]pyridines (3-deazapurines) and their nucleosides as immunosuppressive and antiinflammatory agents. J. Med. Chem. 29: 138-143

Liu, X., Kim, C. N., Yang, J., Jemmerson, R. and Wang, X. (1996) Induction of apoptotic program in cell-free extracts: Requirement for dATP and cytochrome c. Cell 86: 147-157

Liu, X., Zou, H., Slaughter, C. and Wang, X. (1997) DFF, a Heterodimeric protein that functions downstream of caspase3 to trigger DNA fragmentation during apoptosis. Cell 89: 175-184

Mayers, D. L., Mikovits, J. A., Joshi, B., Hewlett, I. K., Estrada, J. S., Wolfe, A. D., Garcia, G. E., Doctor, B. P., Burke, D. S.,
Gordon, R. K., Lane, J. R. and Chiang, P. K. (1995) Antihuman immunodeficiency virus 1 (HIV-1) activities of 3deazaadenosine analogs: increased potency against 3'-azido3'-deoxythymidine-resistant HIV-1 strains. Proc. Natl. Acad. Sci. USA 92: 215-219

Medzihradsky, J. L., Zimmerman, T. P., Wolberg, G. and Elion, G. B. (1982) Immunosuppressive effects of the S-adenosylhomocysteine hydrolase inhibitor, 3-deazaadenosine. J. Immunopharmacol. 4: 29-41

Meyn, R. E., Stephens, L. C., Hunter, N. R. and Milas, L. (1995) Apoptosis in murine tumors treated with chemotherapy agents. Anticancer Drugs 6: 443-450

Nagata, S. (1997) Apoptosis by death factor. Cell 88: 355- 365

Nicholson, D. W. and Thornberry, N. A. (1997) Caspases: killer proteases. Trends Biochem. Sci. 22: 299-306

Prendergast, G. C. (1999) Mechanisms of apoptosis by cMyc. Oncogene 18: 2967-2987

Pritchard, P. H., Chiang, P. K., Cantoni, G. L. and Vance, D. E. (1982) Inhibition of phosphatidylethanolamine $N$-methylation by 3-deazaadenosine stimulates the synthesis of phosphatidylcholine via the CDP-choline pathway. J. Biol. Chem. 257: $6362-6367$

Salvesen, G. S. and Dixit, V. M. (1997) Caspases: Intracellular signaling by proteolysis. Cell 91: 443-446

Shankar, R., de la Motte, C. A. and DiCorleto, P. E. (1992) 3Deazaadenosine inhibits thrombin-stimulated platelet-derived growth factor production and endothelial-leukocyte adhesion molecule-1-mediated monocytic cell adhesion in human aortic endothelial cells. J. Biol. Chem. 267: 9376-9382

Sherman, M. L., Shafman, T. D., Spriggs, D. R. and Kufe, D. W. (1985) Inhibition of murine erythroleukemia cell differentiation by 3-deazaadenosine. Cancer Res. 45: 5830-5834

Thompson, E. B. (1998) The many roles of c-Myc in apoptosis. Annu. Rev. Physiol. 60: 575-600

Thornberry, N. A. and Lazebnik, Y. (1998) Caspases: Enemies Within. Science 281: 1312-1316

Wang, C. Y., Mayo, M. W. and Baldwin, Jr. A. S. (1996) TNFand cancer therapy-induced apoptosis: Potentiation by inhibition of NF-kappaB. Science 274: 784-787

Wiesmann, W. P., Johnson, J. P., Miura, G. A. and Chiang, P. K. (1985) Aldosterone-stimulated transmethylations are linked to sodium transport. Am. J. Physiol. 248: F43-F47

Yang, J., Liu, X., Bhalla, K., Kim, C. N., Ibrado, A. M., Cai, J.,Peng, T-I., Jones, D. P. and Wang, X. (1997) Prevention of apoptosis by Bcl-2: Release of cytochrome c from mitochondria blocked. Science 275: 1129-1132

Zimmerman, T. P., lannone, M. and Wolberg, G. (1984) 3Deazaadenosine. S-adenosylhomocysteine hydrolase-independent mechanism of action in mouse lymphocytes. J. Biol. Chem. 259: 1122-1126

Zimmerman, T. P., Wolberg, G. and Duncan, G. S. (1978) Inhibition of lymphocyte-mediated cytolysis by 3-deazaadenosine: evidence for a methylation reaction essential to cytolysis. Proc. Natl. Acad. Sci. USA 75: 6220-6224 